\title{
IDENTIFICATION OF TWO X-RAY MINIFLARES WITH $\mathrm{H}_{\alpha}$-SUBFLARES
}

\author{
A. Schadee* and V. Gaizauskas** \\ *Laboratory for Space Research, Beneluxlaan 21, 3527 HS \\ Utrecht, The Netherlands \\ ${ }^{* *}$ Herzberg Institute of Astrophysics, National Research Council \\ of Canada, Ottawa, Canada KIA OR6
}

\section{ABSTRACT}

Active regions show many short-lived emissions in the $3.5-5.5 \mathrm{keV}$ range that are 100 to 1000 times weaker than "normal" $\mathrm{X}$-ray flares. The hypothesis that they may well be miniflares is supported by the simultaneous occurrence of $2 \mathrm{H}_{\alpha}$-subflares at the site of weak $\mathrm{X}$-ray sources.

\section{INTRODUCTION}

The low background noise in the lowest energy (3.5 - 5.5 keV) channel of HXIS aboard SMM /1/ allows the detection of very weak $x$-ray sources $/ 2 \%$. There is evidence for two types of sources: long-lived sources (LLS) with lifetimes of more than half an hour and with gradual lightcurves as opposed to short-lived sources (SLS) with lifetimes of 1 to 15 minutes, typically 5 to 10 minutes. SLS's mostly show a peaked maximum and are quite compact $(<1 / 2$ arcmin), in contrast to LIS's, which tend to cover more extended areas (1-3 arcmin).

If at least some of the SLS's are miniflares, i.e. the same phenomenon as "normal" flares on a 100 to 1000 times smaller energy scale and if the $\mathrm{H}_{\alpha}$-response is concordantly smaller, their optical identification meets with severe observational difficulties. Therefore, these observations of the coincidence of SLS's with two $H_{\alpha}$-subflares observed under very good seeing conditions, lends considerable support to the miniflare hypothesis.

\section{OBSERVATIONS}

We studied observations of AR 2456 = Hale region 16850 during the SMM day $17: 36$ - 18:19 UT on 20 may 1980. According to Harvey /3/, this region was decaying and flare activity nearly ceased for two days (20 May inclusive) prior to the major two-ribbon flare of 21 May, 20:54 UT.

\section{i) $x$-rays, $3.5-5.5 \mathrm{kev}$}

Deconvolved HXIS images of its lowest energy band are presented in Figure 1. Figure 1a shows accumulated images of the coarse field of view (FOV), 6' across with pixels of $32^{\prime \prime} x 32^{\prime \prime}$ (FWHM). The fine FOV (Figure 1b), concentric with the coarse FOV, measures 2 ' across with 8 " $x$ 8" pixels. Contourlevels have intervals of a factor 2 from the maximum (dot) downward to the 5-sigma level of the background, which is 0.005 counts/s/pixel. Due to its larger pixel size, the coarse FOV is more sensitive than the fine.

Three sources, A, B and C, are identified. All three are quite compact, the diameter of their half-maximum contours being close to the instrumental FWHM. Only A can be seen in the fine FOV; $B$ is too weak and $C$ lies outside the fine EOV, $1 / 2$ arcmin south of $A$. The lightcurves of their coarse FOV images are given in Figure 1c. A and $C$ have so many pixels in common that their lightcurves had to be combined. However, their independence is evident from the spatial images. The pixels $B$ shared with $A$ and $C$ are omitted from lightcurve $B$ (the counts in this curve prior to $17: 39$ are due to $\mathrm{A}$, as SMM was repointed 38 " westward at that time).

A went through a maximum at $17: 39$ and had almost disappeared by $17: 47$. (the flux in the lightcurve $A+C$ from 17:49 - 18:00 is mainly from $B$, as evidenced by the spatial images). The statistics of $B$ are too poor for establishing a temporal behaviour. Careful analysis shows $x$-ray flux present from $17: 49$ to 18:05. C rises slowly as of 18:00 to a maximum at 18:17.

The $x$-ray fluxes are very low indeed and just barely noticeable in the next higher energy band $(5.5-8 \mathrm{keV})$. From the flux ratio of both bands, integrated over their whole existence 


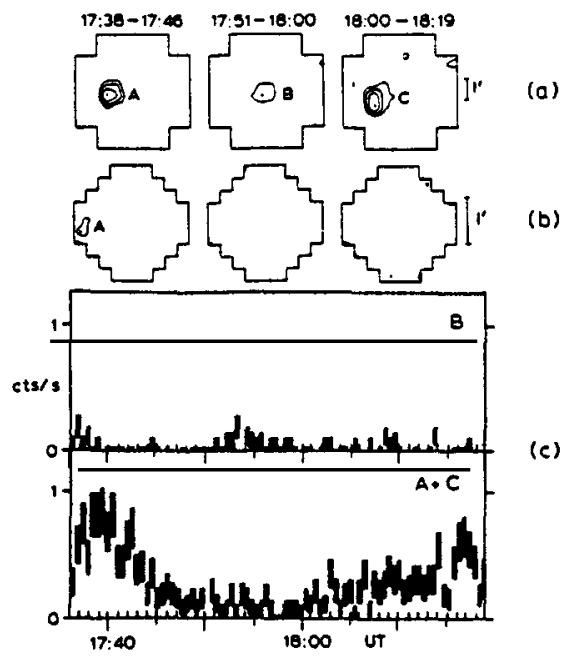

Fig. 1. The X-ray observations, 3.5 - $5.5 \mathrm{keV}$. a) coarse FOV; b) fine Fov; c) lightcurves.

to improve statistics, the following temperatures $T_{6}$ in $M K$ were found: $A 10.6+0.5, B 14.5$ \pm 2.5 and $C 9.4 \pm 0.5$ with emission measures $\int \mathrm{ne}^{2} \mathrm{dV}\left(\mathrm{cm}^{-3}\right)$ at the peak flux: A $\overline{6} \times 10^{46}, \mathrm{~B} 6$ $\bar{x} 10^{45}$ and $c 8 \times 1046$. We stress that these numbers are no more than indicative

\section{ii) H-alpha}

Filtergrams at high spatial resolution of the target region were secured at the ottawa $R i v e r$ Solar Observatory (ORSO) every 25 s during this SMM orbit except for a break from 17:49 17:57 UT. The $H_{\alpha}$ FOV can be registered against the HXIS FOV to within 5 " by reference to contemporal filtergrams on a small scale of the entire disc, also secured at oRSo.

Two impulsive subflares, each consisting of widely separated, compact patches (knots) of $H$ emission (Figure 2) were observed in close coincidence in space and time with two miniature $x-r a y$ bursts at sources $A$ and $C$ (Figure 1). For the first event, the tiny burst $\alpha_{2}$ (Figure 2B), near the eastern border of the HXIS fine FOV, brightened suddenly at 17:35:09 at $H_{\alpha}+$ $0.8 \mathrm{~A}$. By 17:35:12, $\alpha_{1}-\alpha_{3}$ were all brightening and merging; at 17:35:33 another small flaring patch brightened at $\beta$ (Figure 2B), $3.5 \times 10^{4} \mathrm{~km}$ away and just on the opposite side of the large filament which separates magnetic polarities in the active region. Peak brightness occurred around 17:37; the four knots then faded to preflare brightness by 17:49. A fifth knot, $\alpha_{4}$ (Figure 2D), only $8 \times 10^{3} \mathrm{~km}$ from $\alpha_{2}$ on a trajectory towards $\beta$, brightened around $17: 38$ and was still brighter than its pre-flare level when the data break began at 17:49. The X-ray source A of Figure $1 b$ centered its peak emission near $\alpha_{4}$. Either a small surge or loop structure, activated just north of knot $\beta$, grew and seemingly merged with the large filament overlying the line of polarity inversion (Figure 2 , panels $B-G$ ).

For the second event, a different cluster of four $H_{\alpha}$ knots coincide in time and position with HXIS source $C$, just east of the fine FOV. Several bursts along the narrow segment of weak plage at $\delta_{1}$ (Figure $2 G$ ) began to brighten slowly around 18:05 and stayed bright for the remainder of the SMM orbit. Two compact knots, side-by-side at $\delta_{2}, 3 \times 10^{4} \mathrm{~km}$ away from $\delta_{1}$ and on the opposite side of the polarity inversion line, brightened about one minute later. A much more impulsive sequence was initiated at 18:15:09 when patches brightened simultaneously at $\delta_{3}$ and $\delta_{4}, 2.6 \times 10^{4} \mathrm{~km}$ apart. Their brightness peaked 18:16 - 18:17, and was still fading at the end of the SMM day. Meanwhile the filamentary structures between $\delta_{2}$ and $\delta_{3}$ underwent drastic changes. They lie close to the peak emission of $x-r a y$ source $C$ in Figure 1a.

The second subflare appears to be the superposition of two spatially separated events: a gradual event involving $\delta_{1}$ and $\delta_{2} ;$ an impulsive one consisting of $\delta_{3}$ and $\delta_{4}$. Each has simultaneous, or near simultaneous, brightenings in compact areas fixed in magnetic fields of opposite polarity, like the subflare associated with source $A$.

HXIS source $B$ was activated during the data break of $8 \mathrm{~min}$ at ORSO. Its location is identified with a small plage just outside the western boundary of the panels in Figure 2 . The bright core of the plage seen at the end of the data break was roughly double its size when the data break began, about the size of $\alpha_{4}$. In the light of events $A$ and $C$, it is reasonable to conjecture that a similar short-lived chromospheric subflare erupted here in association with the $X-r a y$ miniflare $B$. 


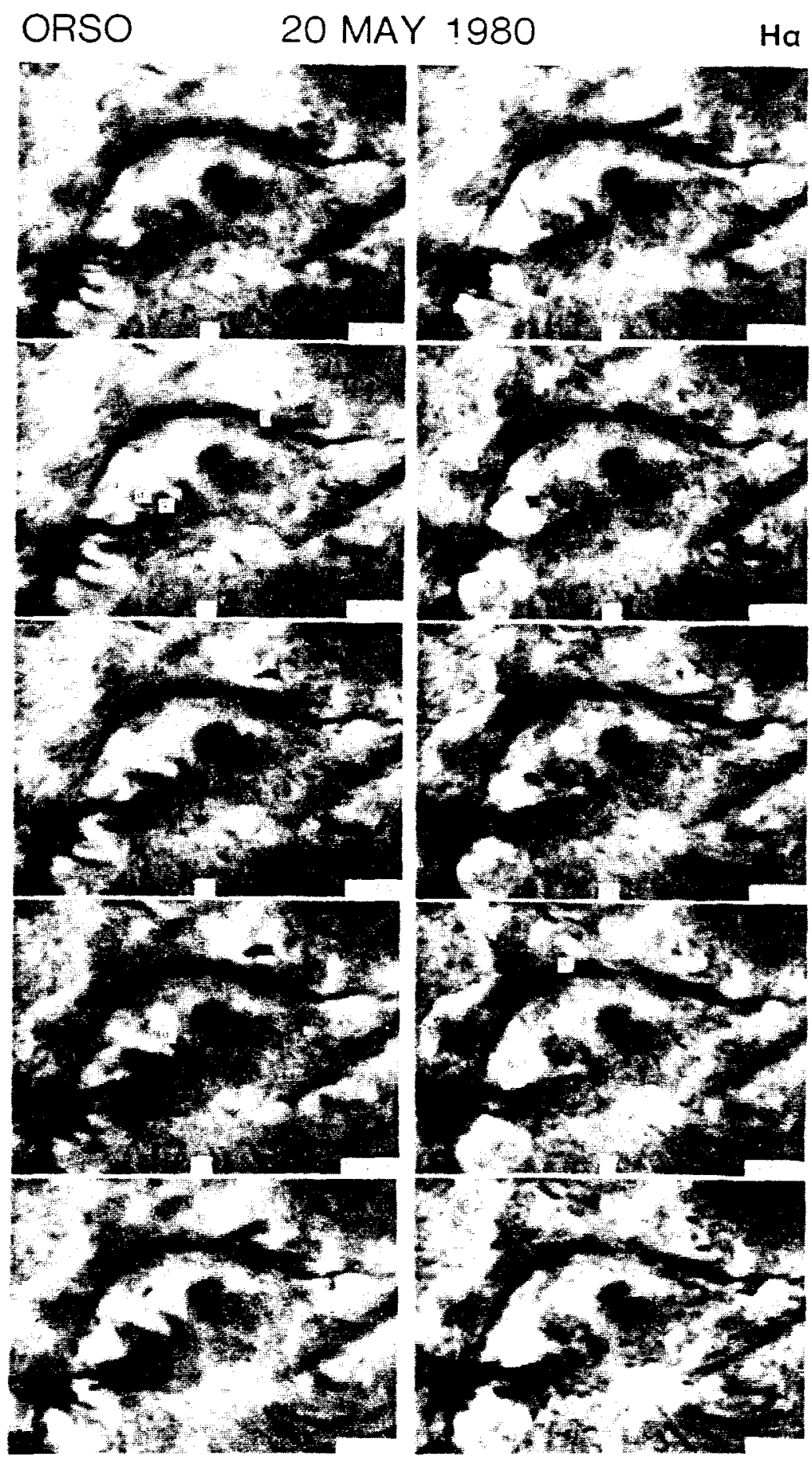

Fig. 2. Time-sequence of $H_{\text {t }}$ filtergrams of a portion (95" $x$ 130") of the full EOV containing AR 2456. The long side of each panel is oriented E-W (celestial), with North at the top, West to the right. UT is shown in the lower right corner of each panel. 


\begin{abstract}
iii) other observations
The Hard X-Ray Burst Spectrometer on board SMM, a monitoring device, noted three single spikes in its lowest energy channel $(27-32 \mathrm{keV})$ at 17:56, 18:06 and 18:10. They seem not related to the region we studied.

The Flat Crystal Spectrometer made heliograms in the lines of O VIII (3), Ne IX (3.5), Mg XI (6) and Si XIII (9.5) at 17:35, 17:37, 17:43, 17:48, 17:54, 17:59 and 18:11 (in brackets the line temperatures $T_{6}$ ). Source $A$, at the east edge of the Fov, is in Si XIII only and barely visible at $17: 37$ as a compact source. Towards lower temperature lines the source becomes more and more extended. The decrease of flux is far slower than in the higher temperaturs regime that HXIS observes. Source $B$, not seen In Si XIII, may be present in O VIII, but here it mixes with the extended source A. Its identification in Ne IX and $\mathrm{Mg} \mathrm{XI}$ is questionable. Source C, finally, lies outside the FCS FOV.
\end{abstract}

The Owens Valley Radio Observatory, observing with a two disk interferometer at 10.5 GHz, reports a subflare at $17: 36: 20$ of 0.12 correlated $s$ fu, 208 circularly polarized. This subflare started shortly after $17: 35$ and lasted less than $90 \mathrm{~s}$.

\title{
DISCUSSION
}

Combining the radio, $\mathrm{X}$-ray and $\mathrm{H}_{\alpha}$ observations, there is no doubt that the impulsive $\mathrm{x}$-ray sources at 17:39 and 18:17 were associated with $H_{\alpha}$-subflares and that in fact some, at least, of the SLS's are miniflares following the flare scenario, but at a much reduced scale. We even see near-simultaneous flaring in patches located in magnetic fields of opposite polarity, as in much larger two-ribbon flares. The simultaneous onset of the radio-subflare with the red-shifted $\mathrm{H}_{\alpha}$-kernel shortly after 17:35 indicates the injection of fast electrons into the low corona and chromosphere, apparently too few for observable hard $x$-rays over $10 \mathrm{keV}$. Following evaporation, a tiny loop lights up in $\mathrm{x}$-rays, peaking some 3 min after the injection of fast electrons.

The data gap of $\mathrm{H}_{a}$-observations from 17:49 till 17:57 prevents a positive correlation between the $x$-rays of source $B$ and low-atmospheric phenomena. However, there are a fe; interesting points to be mentioned: 1) this $x$-ray source was probably hotter than the 17:39 subflare loop, maybe about $14 \mathrm{MK} ; 2$ ) there is no or at best an extremely small event in the FCS heliograms, i.e. in lines ranging from 2-10 MK; 3) nothing noticeable occurred at 10.6 $\mathrm{GH} z$; 4) there is no indication of an impulsive event preceding this very low $\mathrm{X}$-ray flux. The origin of this radiation over this plage region is therefore enigmatic.

\section{ACKNOWLEDGEMENTS}

We gratefully acknowledge Dr. R.B. Bentley (FCS), Dr. B.R. Dennis (HXRBS) and Dr. G. Hurford(OVRO) for providing us with their observations. This collaboration was facilitated by participation in the series of workshops for the Flare Build-up Study and for NASA's Solar Maximum Mission.

\section{REFERENCES}

1. H.F. van Beek, P. Hoyng, B. Lafleur and G.M. Simnett, Solar Phys. 65, 39 (1980)

2. A. Schadee, C. de Jager and z. Svestka, Solar Phys; 89, 287 (1983)

3. J.w. Harvey, Adv. Space Res. 2, \#11, 31 (1983) 